\title{
Permeation of pure gases through silica membranes with controlled pore structures
}

\author{
Berna Topuz*, Muhsin Çiftçioglu \\ Department of Chemical Engineering, İzmir Institute of Technology, Urla-TR, 35430, Urla, Izmir, Turkey \\ email: bernatopuz@iyte.edu.tr
}

Received 18 October 2005; accepted 2 March 2006

\section{Introduction}

The superior thermal/chemical/mechanical stability and the ability of ceramic membranes in affecting the transport rates of chemical species through their processing controllable pore structures make them very attractive for many separation problems. Highly selective microporous silica membranes with high fluxes could be prepared by sol-gel dip coating processes [1]. The structure of the thin silica layer mainly depends on the size and the shape of the silicalite polymers and their packing behavior during drying and heat treatment. Design of the pore networks has a great importance to decide the transport properties through the membrane since permeation and the permselectivity are mainly determined by the microstructure of the membrane such as pore size and distribution, porosity as well as the interaction of permeating species with pore walls [2]. The key to improve the permeability and the permselectivity is the control of the pore structure of the membrane top layer. The permeation rate of different pure gases through the well-defined porous membrane make a clear identification of gas transport mechanisms

*Corresponding author. through these controlled pore-networks. Being able to know the structure of pore network of the membrane that control the gas permeation can reveal more information on how gas transport mechanisms are changing with respect to the change in pore diameter which can be easily controlled by selecting the sphere size in colloidal silica preparation. The use of mono-dispersed silica spheres having a size smaller than $10 \mathrm{~nm}$ with narrow particle size distribution can make possible to prepare microporous silica membranes with controllable pore structures for specific applications in gas separation.

This study aims for a better understanding of how the processing parameters like reactant concentration, reaction temperature, aging time, heat treatment conditions affect the silica membrane pore structure and the permeation of pure gases through the membrane.

\section{Theory and experimental}

Silica sols were synthesised by acid catalysed hydrolysis and condensation of tetraethyl orthosilicate (TEOS) in ethanol. The acid to alkoxide molar ratio was changed from 0.05 to 0.17 in order to investigate the effect of acid concentration on the gas permeation through the 




Fig. 1. Aging time effect on $\mathrm{O}_{2}$ permeability through sol-gel derived silica membranes.

membranes. Aging was performed on silica sol at $50^{\circ} \mathrm{C}$ up to $16 \mathrm{~h}$ to investigate the effect of aging on membrane pore structure. Mono-dispersed silica sols having well-defined silica spheres ranging in size from 200 to $5 \mathrm{~nm}$ were prepared by using Stober process. The effect of ammonia/water concentration and temperature on the particle size of the silica spheres and membrane pore diameter have been investigated by laser light scattering particle size (Malvern, ZetaSizer 3000 HAS) and $\mathrm{N}_{2}$ adsorption-desorption (Micromeritics-ASAP 2010) analysis. The microstructure and thickness of the silica membranes deposited on slip-casted zirconia supports were examined by SEM (Philips, XL30 SFEG). Single gas permeation was carried out for pure gases $\mathrm{O}_{2}, \mathrm{~N}_{2}$ and $\mathrm{CO}_{2}$ by using pressure controlled dead-end mode membrane test system at room temperature. The measured membrane area was $12.5 \mathrm{~cm}^{2}$.

\section{Results and discussion}

An increase in the acid content of the polymeric silica sol increased the porosity (shown by an increase in gas permeance) which consequently reduced the permselectivity of the gases which may be due to the faster condensation reaction during sol formation. The effect of gel aging time on $\mathrm{O}_{2}$ permeation for the 2-layer 50 and $400^{\circ} \mathrm{C}$ treated silica membranes is given in Fig. 1. Permeation rate of $\mathrm{O}_{2}$ through $400^{\circ} \mathrm{C}$ and $50^{\circ} \mathrm{C}$ treated membrane changes significantly with aging time up to $4 \mathrm{~h}$ due to the changes in the pore structure of the membrane with increasing aging time.

The unaged unsupported membrane had a Langmuir surface area of $487 \mathrm{~m}^{2} / \mathrm{g}$ and $\mathrm{H}-\mathrm{K}$ pore diameter of $0.6 \mathrm{~nm}$ and the pore size increased from $0.6 \mathrm{~nm}$ to $0.68 \mathrm{~nm}$ upon increasing the aging time to $3 \mathrm{~h}$ at $50^{\circ} \mathrm{C}$ (Fig. 2).

\section{Conclusions}

The nature of the pore structure is one of the most important factors along with molecular properties and the interaction with the pore
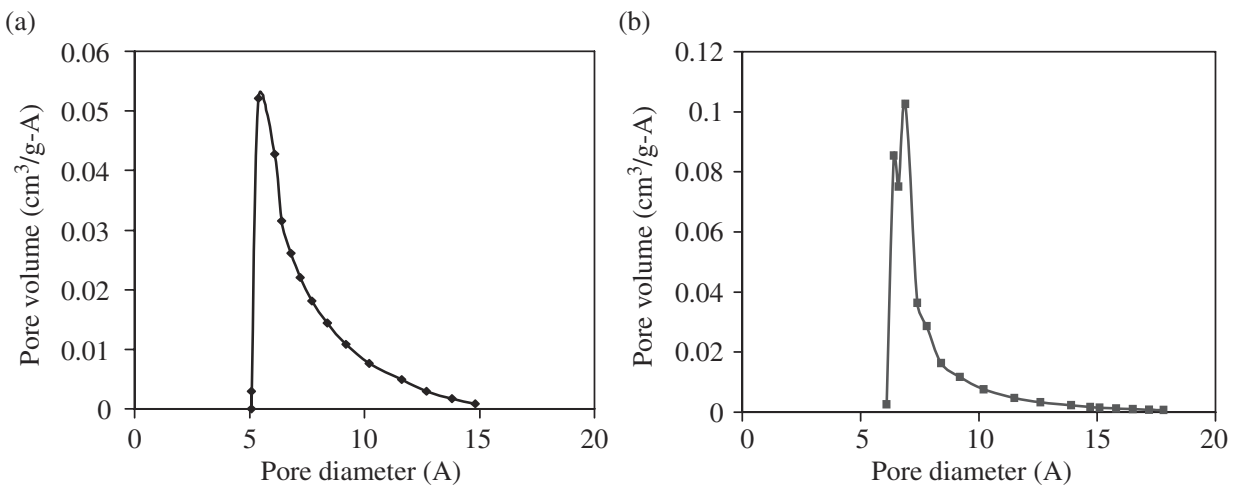

Fig. 2. Pore size distributions of $400^{\circ} \mathrm{C}$ treated unsupported membranes: (a) unaged, (b) 3-h aged. 
walls of gaseous species during their transport through the porous membrane. Thus, it is important to precisely control of the membrane pore size uniformity in nanometer scale without pinholes or cracks. The analysis of the permeation result with controlled membrane structure may lead the more accurate determination of transport mechanisms through porous membrane structures with different pore diameter.

\section{References}

[1] R.M. De Vos and H. Verweij, Improved performance of silica membranes for gas separation, J. Membr. Sci., 143 (1998) 37-51.

[2] J.D.F. Ramsay, Characterization of the pore structure of membranes, MRS Bull., March (1999) 36-40. 